\title{
The business of bioanalysis: new technology integration into bioanalytical workflows
}

\author{
Scott Summerfield*,1, Roger Hayes ${ }^{2}$, Meina Liang ${ }^{3}$, Afshin Safavi ${ }^{4}$, Dominic Warrino ${ }^{5}$, Lina \\ Luo $^{6} \&$ lan Moore ${ }^{7}$ \\ ${ }^{1}$ Bioanalysis, Immunogenicity \& Biomarkers, GlaxoSmithKline Pharmaceuticals, Research \& Development, Park Road, Ware, SG12 \\ ODP, UK \\ ${ }^{2}$ MPI Research, Inc., A Charles River Company, Main Street, Mattawan, MI 49071, USA \\ ${ }^{3}$ Clinical Pharmacology \& DMPK, 121 Oyster Point Boulevard, South San Francisco, CA 94080, USA \\ ${ }^{4}$ BioAgilytix Bioanalysis, 2300 Englert Drive, Durham, NC 27713, USA \\ ${ }^{5}$ KCAS Bioanalytical \& Biomarker Services, 12400 Shawnee Mission Parkway, Shawnee, KS 66216, USA \\ ${ }^{6}$ Department of Pharmacokinetics, Dynamics \& Metabolism, Pfizer, Inc., 445 Eastern Point Road, Groton, CT 06340, USA \\ ${ }^{7}$ Nominal Mass Platforms, SCIEX, 71 Four Valley Drive, Concord, ON, L4K 4V8, Canada \\ *Author for correspondence: scott.g.summerfield@gsk.com
}

First draft submitted: 6 August 2018; Accepted for publication: 11 October 2018; Published online: 23 October 2018

Keywords: bioanalytical platforms • computer system validation • immunogenicity • LC-MS/MS • outsourcing - pharmacokinetics

Scientific conferences offer a venue for stakeholders in the bioanalytical community to converge and discuss current and emerging trends affecting the discipline. In recent years, panel discussions with key opinion leaders, have become a complementary medium to reflect on the direction of travel faced by the business of bioanalysis [1-3]. These discussions have been filmed and then shared with the bioanalytical community via dual media formats; video access through the Bioanalysis Zone website and an accompanying publication [4]. This particular panel discussion was filmed during the 12th Workshop on Recent Issues in Bioanalysis (WRIB; PA, USA, 9-13 April 2018), and focused on the topic of new technology integration into bioanalytical workflows. Three areas were discussed:

- Where the demand is coming from for new technologies to support bioanalytical measurements?

- What barriers are in the way?

- Where the panel felt the emergent opportunities lie?

Contributing panel members came from the Pharma industry, the CRO sector and also vendors. The discussions are summarized below and can be viewed in full on the Bioanalysis Zone website.

\section{Where is the driver for new technologies coming from?}

Bioanalytical platforms are well established within Pharma and CROs to support conventional small molecules $(<500 \mathrm{amu})$ and monoclonal antibody therapeutics. These methodologies have been broadly amenable to the determination of the pharmacokinetics (PK) or immunogenicity of potential therapeutics as well as complementary biomarkers of pharmacodynamics, safety or efficacy, and are focused largely on MS/MS coupled with HPLC and ligand binding assays (LBA). But, the continuing pursuit to better manage or cure diseases has directed more effort toward novel therapeutic modalities. In turn, this is demanding a more extensive arsenal of analytical technologies including some iterations of current platforms (e.g., high-resolution mass spectrometry and mass cytometry $[\mathrm{CyTOF}]$ ) or hybrid approaches (i.e., combination of MS-based detection with immunoprecipitation). Alongside these new technologies there is the continued strive for process improvement and streamlining laboratory workflows. For CROs and Pharma this could be as simple as leveraging automation ranging from a small automated liquid handler doing one thing very effectively through to more end-to-end solutions like the Gyrolab LBA platform. 


\section{Novel modalities \& bioanalytical measurements}

From a Pharma perspective there is a growing need to forward plan in terms of what these new types of drug and therapy mean for bioanalysis, particularly where measurements cross over the threshold from drug discovery into drug development. Requests to support new modalities like gene therapies and chimeric antigen receptor (CAR) $\mathrm{T}$ cells may require assessment of $\mathrm{PK}$ using unconventional approaches as well as pharmacodynamic end points or more functional assay readouts. Other examples of new modalities touched upon were:

- Gene therapy with respect to measurements of biodistribution of the therapeutic agent (e.g., qPCR, positron emission tomography imaging), measurements of the viral or nanoparticle vector (e.g., qPCR, LC-MS), production of a missing protein (LBA) and potential immunogenicity of all of the above (LBA or cell-based assays);

- Oligonucleotides and the need for hybridization techniques (e.g., qPCR or branched DNA) or ultimate sensitivity (tiny amounts of oligonucleotides in tiny amounts of tissue biopsies);

- CAR macrophages and CAR T cells where screening multiple human cell lines would be required in a very iterative process under sterile conditions. Measurements would not likely correspond to conventional PK end points on a discrete analyte, but may involve concentration-time measurements of cells utilizing flow cytometry (or molecular approaches) or testing how the immune system is being augmented and the associated safety. The monitoring of augmentation of immune cells is performed predominately by flow cytometry and elispot platforms;

- Stem cell therapies for enzyme replacement where immunogenicity risks get high. Patients who do take to the therapy will be susceptible to antibodies forming against the de novo enzyme/protein as it will be recognized as a foreign antigen by the patient. Although, typically the therapy for enzyme replacements is intracellular and not circulating, they still require validation of an antienzyme replacement antibody assay. Patients can be exposed via cellular blebs, apoptotic cells, necrotic cells and cell debris/waste.

Since the current outsourcing paradigm continues to favor the majority of regulatory studies being externalized, CROs are at the forefront of client requests to adopt instrumentation originally developed for exploratory research and deploy them in drug development (to aid dose selection, asset progression and translational pharmacology measurements). CRO leaders raised two key points that any dialog with the client should include. First, there is the question on what data are absolutely required for decision making and whether something truly 'out of the box' is needed? Second, there must be a clear awareness that newer technologies may be unproven to regulatory bodies and, hence, there could be risks carried forward on critical aspects like data quality, data comparability and data integrity [5]. Although many buzzwords flutter around new technologies (e.g., multiplexing), the prevalent driver around new technology implementation is still sensitivity (such as, measurements of biomarkers expressed at low basal levels).

\section{Demands for increased sensitivity remains a major focus}

Although molecular novelty is increasing across the overall portfolios of Pharma and Biotech companies, small molecules and conventional monoclonal antibody still comprise a substantial portion of current assets in drug development. Continued improvements in the sensitivity of LC-MS technologies for small molecule bioanalysis are still seen as desirable because laboratories do not have to labor as hard to develop and then validate assays to generate quality results during production. Enhanced sensitivity allows for smaller sample volumes or a simpler extraction procedure to dilute out matrix effects, thereby simplifying workflows.

For LBA technologies greater sensitivity would still be of value to extend or simplify the quantitation possibilities for biomarkers. In the discovery arena, small sample volumes become important when trying to use the same sample for both PK and pharmacodynamic measurements; for example, in preclinical models of disease using genetically engineered animal models. Another example discussed was for antibody-drug conjugates for oncology targets where a relatively small tumor biopsy is used for multiple measurements. Increased sensitivity was also felt to be crucial for new formulations, such as nanoparticles, where measuring both total and free releases would be needed to justify safety and efficacy.

\section{The technology intersection for Pharma, CROs \& vendors}

Today, pharma/biotech organizations focus on the majority of their internal bioanalytical resources toward drug discovery and early stages of drug development, while externalizing many later stage activities to CROs. For many 
pharma companies, laboratories and staff training are no longer configured to support much regulated bioanalytical work internally, if at all. CROs (in their capacity as a resource partner for drug development) have many things to do when trying to introduce new technologies; learn how to operate the systems, validate them and then get workflows efficient (all in a short space of time). There is almost an implicit expectation that CROs already have that technology up and running, ready and waiting. The startup costs for new technologies, such as ultrasensitive equipment, can deter clients and promote a kind of reverse engineering where the capabilities of an existing technology can be developed to meet the immediate need.

Equally for instrument vendors, thinking ahead needs to become an industry-wide trait in advance of novel modalities entering drug development and requiring regulated bioanalytical support. Furthermore, the emerging paradigm of translational medicine also demands that critical measurements originating in drug research are transitioned into clinic development. Some vendors are indeed looking proactively at futureproofing their platforms to ensure robust data are at the standard required for use in regulated study support, including the software perspective. Examples would be instrument manufacturers with some technology presence already in regulated bioanalysis (LC-MS or LBA vendors). It was noted that research instrumentation might need a creative solution to software validation or adding automation to make them amenable for the latter stages of drug development.

For very practical reasons, customer alignment is crucial for instrument vendors. The translational medicine approach also requires vendors to understand what problems need solving in the clinical setting. In the field of MS, understanding customer needs around sensitivity and improved signal-to-noise has necessitated looking beyond the mainstay of tandem quadrupole technology toward accurate mass or an orthogonal technique such as ion mobility. But with increased complexity, vendors are also hearing the call from CROs around usability, ease of learning, ease of maintenance and then also purchase/running costs. The latter becomes important for CROs because of the risks associated with only having a single system and no redundancy.

\section{What are the barriers to driving new technologies into bioanalysis?}

Barriers to the introduction of new technologies were identified around training, cost, uncertainty of sufficient utilization, return on investment (ROI), vendor input and regulatory.

With respect to training barriers, there was an observation that Pharma continues a trend of recruiting from $\mathrm{CROs}$ although the reverse situation has become more common place. The current generation of young scientists is also more forward, mobile and keen to accelerate their development through rapid career moves. Conversely for the business of bioanalysis, return on the training investment is needed before staff move on, which is placing new pressure on the CRO sector as the major talent pool in today's marketplace.

There was general agreement that cost (in terms of price tag) of a new analytical platform is only a small part of the overall investment; time/cost of implementation, developing the interface with an laboratory information management system (LIMS), amendment of standard operation procedures (SOPs), training and maintenance contracts are all contributors. In today's increasingly regulated climate, it is important to adequately manage the entire GLP/GCP Clinical Labs risks (not just lab workflows) and demonstrate acceptable data integrity. Computer system validation was noted to be a major challenge, perhaps taking 6 months to complete even though most instrumentation is likely commissioned within a month. On this front, it was felt that a lot of vendors are not yet ready for prime time, while CROs sit on the front line trying to get them to understand what is needed to meet 21CFR11 compliance and future data integrity requirements. Established vendors do look to plan ahead (e.g., major players in the MS and LBA markets), but with more exploratory instrumentation leaking into the regulated environment there is a cause for concern. Although a vendor's computer system validation package provides a useful baseline, the current regulatory environment requires many aspects to be completed again in situ to demonstrate suitability within the testing facility. Some CROs have begun to pre-empt purchase; for example, demo the software as well as the instrument to ascertain how long the internal loading will be to complete software validation. Notwithstanding this, overall the advice from the panel members to vendors was to ensure their development of computer software and hardware not only provided intuitive user tools, but also that data integrity, audit trails, data capture and archive can enable the transition from research to drug development.

The landscape around ROI has also evolved substantially. During the late 1990s when the bioanalytical support was still centralized within Pharma, funding was looser and instruments were purchased as much for their potential and having a new technology available for putative future need. Today, regulatory requirements are stricter, funding is tighter and Pharma has divested many regulated activities to CROs. This means industry must think harder on what new platforms to invest in; for example, for the CRO sector, are there sufficient clients and a solid partnership 
for future work? CROs are seeing many buzzwords included in request for information packs but the flip side is the long game and building confidence around what technology has the potential to become an industry standard. In addition, proof of concept data from vendors is very important, especially in the run up to introducing new measurement platforms. The field of LC-MS quantitation was viewed as an area where this is successful and interactions are collaborative.

\section{Where are the opportunities?}

The panel discussed what technologies were likely to mature in both Pharma and CROs, what could tip the ROI for being positive and avenues to engage vendors and regulators?

After a long incubation period, high-resolution MS is most likely to see a more widespread adoption as a quantitative bioanalytical tool. Partly this is because the technology already has a foothold in some applications but also because software development has advanced alongside the more traditional low-resolution MS/MS platforms.

Mass cytometry is an exciting development although, at a current cost of approximately $\$ 500,000$ for a system, this is likely to be a longer term proposition for CROs and there is an associated need for MS expertise [6,7].

Realizing a suitable ROI means that destructive technologies are unlikely to be introduced at CROs and an immediate need would be most important. For any new technology, robustness is critical because clinical trial support may last several years and data must be reproducible, reliable and defendable. There is also an element of 'what came first, the chicken or the egg?' because CROs need the surety of business; so, the ROI tipping point is arguably partnership. Transactional relationship between Pharma and CROs is unlikely to develop sufficient trust for new technology implementation. As more scientists move into procurement groups, there continues to be improved dialog around the need for advanced technology at the $\mathrm{CRO}$ while also an understanding of the inherent costs of its implementation.

Regulatory creep and implementing totally new technology mid stream was viewed as very risky. What would Pharma's appetite for a CRO changing to better instrumentation be with the risk of spotting something that was not noticed before? Surveys and output from panel discussion should help vendors understand unmet needs in Pharma and then prioritize development and introduction of new platforms. Also, the evolution of conferences such as Workshop on Recent Issues in Bioanalysis to include regulatory authorities alongside current science has certainly enabled better dialog.

\section{Conclusion}

New modalities and the continued need for increased sensitivity are driving the development of many new technologies. In concert, there is a strong industry ambition to deliver better translational end points from the research and clinical development phases. This is pushing technologies originally conceived for discovery research activities toward the regulated world to complement the measurements from HPLC-MS/MS and LBA. The current outsourcing paradigm sees CRO at the forefront of this technology onslaught into drug development; however, the burden is carried between Pharma, CROs and instrument vendors. Partnership and looking forward toward the clinical setting are most likely to enable the optimum implementation of new technologies for the next generation of therapeutics. As an industry we need to remain inventive, extending (where possible) the applicability of established technologies that are already accepted by regulatory bodies, but be innovative highlighting the unmet need in current technologies early so that the transition from drug research to drug development is seamless.

\section{Future perspective}

Panel members were asked what would be of value in the future. On software validation, having applications available in the cloud by a vendor, regulators could be happy that the instrument is used in the way vendors had intended and, therefore, eliminate or significantly reduce the burden of on-site validation. Although the activation barrier is very high to achieve this, the implementation of new instrumentation would be more streamlined and therefore benefit drug development trials and ultimately patients earlier.

For those working with protein-based therapeutics, solving drug tolerance challenges for immunogenicity assessment was seen as an important development for the next generation of technologies and innovative assay approaches.

One wish was to evolve more toward 'plug and play' bioanalysis because drug development is likely to be spread even more across the world with mobility of multilingual scientists trained in several countries. Ensuring robustness would be a critical feature. 
Wider development of flow cytometry was seen as a valuable future horizon for new modalities. But today it was felt that expertise is limited, official regulatory guidance is yet to emerge (although White Papers are available [8-11]), while the future is likely to require validation to GLP standards. The elispot platform has even fewer experienced CROs and no white papers or guidance documents.

There is a strong desire to see continued simplification of software and user interfaces alongside the old favorites of raw sensitivity, robustness, lower barriers to validation, speed of processing, ease of processing, data handling, ease to share and store data.

\section{Acknowledgements}

The authors would like to thank D Murray, N Maundrell and S Nadarajah (employees of Future Science Group) for facilitating the round table discussion and helping to make it such a positive and enjoyable event.

\section{Financial \& competing interests disclosure}

The authors have no relevant affiliations or financial involvement with any organization or entity with a financial interest in or financial conflict with the subject matter or materials discussed in the manuscript. This includes employment, consultancies, honoraria, stock ownership or options, expert testimony, grants or patents received or pending, or royalties.

No writing assistance was utilized in the production of this manuscript.

\section{References}

1. Spooner N, Cape S, Hayes R et al. Issues facing the bioanalytical community: summary of round table discussions. Bioanalysis 8(21), 2189-2193 (2016).

2. Spooner N, Anderson M, Dillen L et al. The changing world of bioanalysis: summary of panel discussions. Bioanalysis 9(15), 1175-1179 (2017).

3. Spooner N, Beaver C, Kolman J et al. The business of bioanalysis: summary of panel discussions. Bioanalysis 10(15), 1169-1175 (2018).

4. Bioanalysis Zone Panel discussion: new technology integration in bioanalytical workflows. https://www.bioanalysis-zone.com/2018/07/03/panel-discussion-new-tech_bob3/

5. Medicines \& Healthcare Products Regulatory Agency. 'GXP' data integrity guidance and definitions. Revision: 1 March 2018. https://mhrainspectorate.blog.gov.uk/2018/03/09/mhras-gxp-data-integrity-guide-published/

6. Bendall SC, Nolan GP, Roederer M, Chattopadhyay PK. A deep profiler's guide to cytometry. Trends Immunol. 33(7), 323-332 (2012).

7. Bandura DR, Baranov VI, Ornatsky OI et al. Mass cytometry: technique for real time single cell multitarget immunoassay based on inductively coupled plasma time-of-flight mass spectrometry. Anal. Chem. 81(16), 6813-6822 (2009).

8. Sommer U, Morales J, Groenewegen A et al. Implementation of highly sophisticated flow cytometry assays in multicenter clinical studies: considerations and guidance. Bioanalysis 7(10), 1299-1311 (2015).

9. Du L, Grover A, Ramanan S, Litwin V. The evolution of guidelines for the validation of flow cytometric methods. Int. J. Lab. Hematol. 37(S1), 3-10 (2015).

10. Brown L, Green CL, Jones $\mathrm{N}$ et al. Recommendations for the evaluation of specimen stability for flow cytometric testing during drug development. J. Immunol. Methods 418, 1-8 (2015).

11. Keeney M, Oldaker T. Flow cytometry CLIA compliance manual International Society for Advancement of Cytometry and Clinical Cytometry Society (2nd Edition). International Society for Advancement of Cytometry (2009). http://www.access.gpo.gov/nara/cfr/waisidx_04/42cfr493_04.html 
